\title{
Lone-Pair Orbital Interactions in Polythiaadamantanes
}

\author{
Joseph E. Norton, Alejandro L. Briseno, Fred Wudl, and K. N. Houk ${ }^{*}$ \\ Contribution from the Department of Chemistry and Biochemistry, University of \\ California, Los Angeles, California 90095-1569
}

Supporting Information

\section{Computational Details, Methodology, and Theory}

DFT. All calculations were carried out using the Gaussian 03 suite of programs. The hybrid density functional Becke3LYP ${ }^{1,2}$ with the 6-31G(d) and 6-311+G(d,p) basis sets were used for all DFT calculations.

HF/6-31G(d) Single-Point Energy Calculations. HF/6-31G(d) single-point energy calculations were performed on B3LYP/6-31G(d) optimized structures. The HF/631G(d) energies are reported in Tables S1 and S2 for comparison with DFT orbital energies reported in the main text.

* Corresponding author. Mailing address: University of California, Los Angeles, Department of Chemistry and Biochemistry, 607 Charles E. Young Drive East, Box 951569, Los Angeles, CA 90095-1569.

Telephone: 310-206-0515. Fax: 310-206-1843. E-mail: houk@chem.ucla.edu. 
Table S1. HF/6-31G(d)//B3LYP/6-31G(d) Orbital Splitting Energies (eV) of Hydrogen Sulfide Model Systems.

\begin{tabular}{|c|c|c|c|c|c|c|c|}
\hline & & (au) & $(\mathrm{eV})$ & & & (au) & $(\mathrm{eV})$ \\
\hline $2_{\mathrm{SH} 2}$ & $\mathrm{E}\left(\mathrm{n}_{\text {номо }}\right)$ & -0.38413 & -10.45 & $9_{\mathrm{SH} 2}$ & $\mathrm{E}\left(\mathrm{n}_{\text {номо }}\right)$ & -0.34893 & -9.49 \\
\hline \multirow[t]{3}{*}{$3_{\mathrm{SH} 2}$} & $\mathrm{E}\left(\mathrm{n}_{\text {номо }}\right)$ & -0.39301 & -10.69 & & $\mathrm{E}\left(\mathrm{n}_{\text {HOMO-1 }}\right)$ & -0.40157 & -10.93 \\
\hline & $\mathrm{E}\left(\mathrm{n}_{\text {номо-1 }}\right)$ & -0.39301 & -10.69 & & $\mathrm{E}\left(\mathrm{n}_{\text {HOMO-2}}\right)$ & -0.44572 & -12.13 \\
\hline & $\mathrm{E}(\mathrm{n})_{\mathrm{Av}}$ & -0.39301 & -10.69 & & $\mathrm{E}(\mathrm{n})_{\mathrm{Av}}$ & -0.39945 & -10.87 \\
\hline \multirow[t]{4}{*}{$4_{\mathrm{SH} 2}$} & $\mathrm{E}\left(\mathrm{n}_{\text {номо }}\right)$ & -0.36357 & -9.89 & & $\beta_{\mathrm{TS}}$ & -0.02420 & -0.66 \\
\hline & $\mathrm{E}\left(\mathrm{n}_{\text {номо-1 }}\right)$ & -0.41619 & -11.33 & $10_{\mathrm{SH} 2}$ & $\mathrm{E}\left(\mathrm{n}_{\text {номо }}\right)$ & -0.36074 & -9.82 \\
\hline & $E(n)_{A v}$ & -0.38988 & -10.61 & & $\mathrm{E}\left(\mathrm{n}_{\text {номо-1}}\right)$ & -0.36794 & -10.01 \\
\hline & $\beta_{\mathrm{TS}}$ & -0.02631 & -0.72 & & $\mathrm{E}\left(\mathrm{n}_{\text {номо-2}}\right)$ & -0.40043 & -10.90 \\
\hline \multirow[t]{4}{*}{$5_{\mathrm{SH} 2}$} & $\mathrm{E}\left(\mathrm{n}_{\text {номо }}\right)$ & -0.34983 & -9.52 & & $\mathrm{E}\left(\mathrm{n}_{\text {номо-3}}\right)$ & -0.43030 & -11.71 \\
\hline & $\mathrm{E}\left(\mathrm{n}_{\text {номо-1 }}\right)$ & -0.40802 & -11.10 & & $\mathrm{E}\left(\mathrm{n}_{\text {номо-4}}\right)$ & -0.43532 & -11.85 \\
\hline & $\mathrm{E}(\mathrm{n})_{\mathrm{Av}}$ & -0.38863 & -10.58 & & $\mathrm{E}(\mathrm{n})_{\mathrm{Av}}$ & -0.39895 & -10.86 \\
\hline & $\beta_{\mathrm{TS}}$ & -0.01941 & -0.53 & & $\beta_{\mathrm{TS}}$ & -0.01712 & -0.47 \\
\hline \multirow[t]{4}{*}{$6_{\mathrm{SH} 2}$} & $\mathrm{E}\left(\mathrm{n}_{\text {номо }}\right)$ & -0.37426 & -10.18 & $11_{\mathrm{SH} 2}$ & $\mathrm{E}\left(\mathrm{n}_{\text {номо }}\right)$ & -0.37184 & -10.12 \\
\hline & $\mathrm{E}\left(\mathrm{n}_{\text {номо-1 }}\right)$ & -0.44563 & -12.13 & & $\mathrm{E}\left(\mathrm{n}_{\text {номо-1}}\right)$ & -0.43868 & -11.94 \\
\hline & $\mathrm{E}(\mathrm{n})_{\mathrm{Av}}$ & -0.39808 & -10.83 & & $\mathrm{E}(\mathrm{n})_{\mathrm{Av}}$ & -0.40526 & -11.03 \\
\hline & $\beta_{\text {TS }}$ & -0.02380 & -0.65 & & $\beta_{\mathrm{TS}}$ & -0.01672 & -0.45 \\
\hline \multirow[t]{5}{*}{$7_{\mathrm{SH} 2}$} & $\mathrm{E}\left(\mathrm{n}_{\text {номо }}\right)$ & -0.35985 & -9.79 & & & & \\
\hline & $\mathrm{E}\left(\mathrm{n}_{\text {номо-1 }}\right)$ & -0.39755 & -10.82 & & & & \\
\hline & $\mathrm{E}\left(\mathrm{n}_{\text {номо-2}}\right)$ & -0.43373 & -11.80 & & & & \\
\hline & $E(n)_{A v}$ & -0.39704 & -10.80 & & & & \\
\hline & $\beta_{\text {TS }}$ & -0.02612 & -0.71 & & & & \\
\hline \multirow[t]{6}{*}{$8_{\mathrm{SH} 2}$} & $\mathrm{E}\left(\mathrm{n}_{\text {номо }}\right)$ & -0.35925 & -9.78 & & & & \\
\hline & $\mathrm{E}\left(\mathrm{n}_{\text {номо-1 }}\right)$ & -0.37799 & -10.29 & & & & \\
\hline & $\mathrm{E}\left(\mathrm{n}_{\text {номо-2}}\right)$ & -0.41697 & -11.35 & & & & \\
\hline & $\mathrm{E}\left(\mathrm{n}_{\text {номо-3}}\right)$ & -0.43829 & -11.93 & & & & \\
\hline & $E(n)_{A v}$ & -0.39813 & -10.83 & & & & \\
\hline & $\beta_{\mathrm{TS}}$ & -0.01963 & -0.53 & & & & \\
\hline
\end{tabular}


Table S2. HF/6-31G(d)//B3LYP/6-31G(d) Orbital Splitting Energies (eV) of Adamantane and Polythiaadamantanes.

\begin{tabular}{|c|c|c|c|c|c|c|c|}
\hline & & (au) & $(\mathrm{eV})$ & & & $(\mathrm{au})$ & $(\mathrm{eV})$ \\
\hline 2 & $\mathrm{E}\left(\mathrm{n}_{\text {номо }}\right)$ & -0.32406 & -8.82 & 9 & $\mathrm{E}\left(\mathrm{n}_{\text {номо }}\right)$ & -0.32313 & -8.79 \\
\hline \multirow[t]{7}{*}{3} & $\mathrm{E}\left(\mathrm{n}_{\text {номо }}\right)$ & -0.33447 & -9.10 & & $\mathrm{E}\left(\mathrm{n}_{\text {HOMO-1 }}\right)$ & -0.34886 & -9.49 \\
\hline & $\mathrm{E}\left(\mathrm{n}_{\text {номо-1}}\right)$ & -0.33447 & -9.10 & & $\mathrm{E}\left(\mathrm{n}_{\text {номо-2}}\right)$ & -0.37454 & -10.19 \\
\hline & $E(n)_{A v}$ & -0.33447 & -9.10 & & $E(n)_{A v}$ & -0.34885 & -9.49 \\
\hline & $E\left(n_{N B O}\right)$ & -0.33932 & -9.23 & & $E\left(n_{N B O}\right)$ & -0.35614 & -9.69 \\
\hline & $\Delta_{\mathrm{TS}+\mathrm{TB}}$ & 0.00000 & 0.00 & & $\Delta_{\mathrm{TS}+\mathrm{TB}}$ & 0.02573 & 0.70 \\
\hline & $\Gamma_{\mathrm{TB}}\left(\mathrm{n}_{\mathrm{HOMO}}\right)$ & 0.00485 & 0.13 & & $\Delta_{\mathrm{TS}+\mathrm{TB}^{\prime}}$ & 0.02568 & 0.70 \\
\hline & $\Gamma_{\text {тв }}\left(\mathrm{n}_{\text {номо-1 }}\right)$ & 0.00485 & 0.13 & & $\Gamma_{\mathrm{TB}}\left(\mathrm{n}_{\text {номо }}\right)$ & -0.01963 & -0.53 \\
\hline \multirow[t]{7}{*}{4} & $\mathrm{E}\left(\mathrm{n}_{\text {номо }}\right)$ & -0.31980 & -8.70 & & $\Gamma_{\text {тв }}\left(\mathrm{n}_{\text {Hомо-1 }}\right)$ & 0.00728 & 0.20 \\
\hline & $\mathrm{E}\left(\mathrm{n}_{\text {номо-1}}\right)$ & -0.34449 & -9.37 & & $\Gamma_{\text {TВ }}\left(n_{\text {HOMO-2 }}\right)$ & 0.02575 & 0.70 \\
\hline & $E(n)_{A v}$ & -0.33215 & -9.04 & 10 & $\mathrm{E}\left(\mathrm{n}_{\text {номо }}\right)$ & -0.33578 & -9.14 \\
\hline & $E\left(n_{N B O}\right)$ & -0.34558 & -9.40 & & $\mathrm{E}\left(\mathrm{n}_{\text {номо-1}}\right)$ & -0.33987 & -9.25 \\
\hline & $\Delta_{\mathrm{TS}+\mathrm{TB}}$ & 0.02469 & 0.67 & & $\mathrm{E}\left(\mathrm{n}_{\text {HOMO-2}}\right)$ & -0.35656 & -9.70 \\
\hline & $\Gamma_{\text {тв }}\left(\mathrm{n}_{\text {номо }}\right)$ & -0.00053 & -0.01 & & $\mathrm{E}\left(\mathrm{n}_{\text {номо-3}}\right)$ & -0.36972 & -10.06 \\
\hline & $\Gamma_{\text {тв }}\left(n_{\text {HOMO-1 }}\right)$ & 0.02740 & 0.75 & & $\mathrm{E}\left(\mathrm{n}_{\text {HOMO-4}}\right)$ & -0.37517 & -10.21 \\
\hline \multirow[t]{7}{*}{5} & $\mathrm{E}\left(\mathrm{n}_{\text {номо }}\right)$ & -0.31886 & -8.68 & & $E(n)_{A v}$ & -0.35542 & -9.67 \\
\hline & $\mathrm{E}\left(\mathrm{n}_{\text {номо-1 }}\right)$ & -0.34903 & -9.50 & & $E\left(n_{N B O}\right)$ & -0.36755 & -10.00 \\
\hline & $E(n)_{A v}$ & -0.33900 & -9.22 & & $\Delta_{\mathrm{TS}+\mathrm{TB}}$ & 0.00409 & 0.11 \\
\hline & $E\left(n_{N B O}\right)$ & -0.34771 & -9.46 & & $\Delta_{\mathrm{TS}+\mathrm{TB}^{\prime}}$ & 0.01669 & 0.45 \\
\hline & $\Delta \mathrm{TS}+\mathrm{TB}$ & 0.03024 & 0.82 & & $\Delta \mathrm{TS}+\mathrm{TB} "$ & 0.01316 & 0.36 \\
\hline & $\Gamma_{\text {тв }}\left(\mathrm{n}_{\text {номо }}\right)$ & -0.00995 & -0.27 & & $\Delta_{\mathrm{TS}+\mathrm{TB}}{ }^{\prime \prime}$ & 0.00545 & 0.15 \\
\hline & $\Gamma_{\text {тв }}\left(n_{\text {Hомо-1 }}\right)$ & 0.01807 & 0.49 & & $\Gamma_{\mathrm{TB}}\left(\mathrm{n}_{\mathrm{HOMO}}\right)$ & -0.00793 & -0.22 \\
\hline \multirow[t]{7}{*}{6} & $\mathrm{E}\left(\mathrm{n}_{\text {номо }}\right)$ & -0.33086 & -9.00 & & $\Gamma_{\text {тв }}\left(\mathrm{n}_{\text {номо-1}}\right)$ & -0.00482 & -0.13 \\
\hline & $\mathrm{E}\left(\mathrm{n}_{\text {номо-1}}\right)$ & -0.35926 & -9.78 & & $\Gamma_{\text {тв }}\left(\mathrm{n}_{\text {номо- }}\right)$ & 0.01099 & 0.30 \\
\hline & $E(n)_{A v}$ & -0.34035 & -9.26 & & $\Gamma_{\text {тв }}\left(\mathrm{n}_{\text {номо-3 }}\right)$ & 0.02770 & 0.75 \\
\hline & $E\left(n_{N B O}\right)$ & -0.36708 & -9.99 & & $\Gamma_{\text {TВ }}\left(\mathrm{n}_{\text {HOMO-4}}\right)$ & 0.02727 & 0.74 \\
\hline & $\Delta_{\mathrm{TS}+\mathrm{TB}}$ & 0.02840 & 0.77 & 11 & $\mathrm{E}\left(\mathrm{n}_{\text {номо }}\right)$ & -0.34774 & -9.46 \\
\hline & $\Gamma_{\mathrm{TB}}\left(\mathrm{n}_{\text {номо }}\right)$ & 0.01240 & 0.34 & & $\mathrm{E}\left(\mathrm{n}_{\text {HOMO-1 }}\right)$ & -0.37755 & -10.27 \\
\hline & $\Gamma_{\text {тв }}\left(n_{\text {Hомо-1 }}\right)$ & 0.05537 & 1.51 & & $E(n)_{A v}$ & -0.36265 & -9.87 \\
\hline \multirow[t]{10}{*}{7} & $\mathrm{E}\left(\mathrm{n}_{\text {номо }}\right)$ & -0.32328 & -8.80 & & $E\left(n_{N B O}\right)$ & -0.37259 & -10.14 \\
\hline & $\mathrm{E}\left(\mathrm{n}_{\text {номо-1}}\right)$ & -0.34197 & -9.31 & & $\Delta_{\mathrm{TS}}+\mathrm{TB}$ & 0.02982 & 0.81 \\
\hline & $\mathrm{E}\left(\mathrm{n}_{\text {номо-2}}\right)$ & -0.35847 & -9.75 & & $\Gamma_{\text {тв }}\left(\mathrm{n}_{\text {номо }}\right)$ & -0.00858 & -0.23 \\
\hline & $E(n)_{A v}$ & -0.34124 & -9.29 & & $\Gamma_{\text {тв }}\left(\mathrm{n}_{\text {HOMO-1}}\right)$ & 0.02846 & 0.77 \\
\hline & $E\left(n_{N B O}\right)$ & -0.35119 & -9.56 & & & & \\
\hline & $\Delta_{\mathrm{TS}+\mathrm{TB}}$ & 0.01869 & 0.51 & & & & \\
\hline & $\Delta_{\mathrm{TS}}+\mathrm{TB}^{\prime}$ & 0.01650 & 0.45 & & & & \\
\hline & $\Gamma_{\text {тв }}\left(\mathrm{n}_{\text {номо }}\right)$ & -0.00979 & -0.27 & & & & \\
\hline & $\Gamma_{\text {тв }}\left(n_{\text {Hомо-1 }}\right)$ & 0.00922 & 0.25 & & & & \\
\hline & $\Gamma_{\mathrm{TB}}\left(\mathrm{n}_{\text {HOMO-2}}\right)$ & 0.02890 & 0.79 & & & & \\
\hline \multirow[t]{13}{*}{8} & $\mathrm{E}\left(\mathrm{n}_{\text {номо }}\right)$ & -0.32898 & -8.95 & & & & \\
\hline & $\mathrm{E}\left(\mathrm{n}_{\text {номо-1 }}\right)$ & -0.33866 & -9.22 & & & & \\
\hline & $\mathrm{E}\left(\mathrm{n}_{\text {номо-2}}\right)$ & -0.35872 & -9.76 & & & & \\
\hline & $\mathrm{E}\left(\mathrm{n}_{\text {номо-3 }}\right)$ & -0.36569 & -9.95 & & & & \\
\hline & $E(n)_{A v}$ & -0.34801 & -9.47 & & & & \\
\hline & $E\left(n_{N B O}\right)$ & -0.36481 & -9.93 & & & & \\
\hline & $\Delta_{\mathrm{TS}+\mathrm{TB}}$ & 0.00968 & 0.26 & & & & \\
\hline & $\Delta_{\mathrm{TS}}+\mathrm{TB}^{\prime}$ & 0.02006 & 0.55 & & & & \\
\hline & $\Delta_{\mathrm{TS}}+\mathrm{TB}^{\prime \prime}$ & 0.00697 & 0.19 & & & & \\
\hline & $\Gamma_{\text {тв }}\left(\mathrm{n}_{\text {номо }}\right)$ & -0.00305 & -0.08 & & & & \\
\hline & $\Gamma_{\text {тв }}\left(n_{\text {Hомо-1 }}\right)$ & 0.00601 & 0.16 & & & & \\
\hline & $\Gamma_{\text {тв }}\left(\mathrm{n}_{\text {номо-2}}\right)$ & 0.02493 & 0.68 & & & & \\
\hline & $\Gamma_{\text {TВ }}\left(\mathrm{n}_{\text {номо-3 }}\right)$ & 0.03928 & 1.07 & & & & \\
\hline
\end{tabular}


Hückel Model for Thiaadamantanes. The purpose of Hückel analysis is to study the relationship between geometry and electronic structure for relatively complex systems, thiaadamantanes. The Hückel model used to describe thiaadamantanes operates under essentially the same assumptions as traditional Hückel molecular orbital (HMO) theory for conjugated $\pi$ systems. The basis set is formed entirely from sulfur lone-pair orbitals, one per atom, instead of parallel p orbitals. The overlap matrix is defined by $S_{i j}=\delta_{i j}$ where overlap of a lone-pair orbital with itself is unity and that between any two nonoverlapping lone-pair orbitals is zero. The $H_{i i}$ matrix elements are set equal to the average orbital energy of the lone pairs in a given thiaadamantane. This energy is defined as $\alpha$ and is negative. The $H_{i j}$ matrix elements between sulfur lone pairs connected by a single carbon are assigned stabilization energy of $\beta$, where $\beta$ is also negative. Matrix elements $H_{i j}$ for distally located sulfurs are set equal to zero. For the purpose of simplifying calculation of the determinant, $X=\frac{\alpha-E}{\beta}$.

The following determinates for thiaadamantanes obtained from the equation

$$
\left|\begin{array}{ccc}
A_{11} & \cdots & A_{1 i} \\
\vdots & \vdots & \vdots \\
A_{i 1} & \cdots & A_{i i}
\end{array}\right|=0 \text {, }
$$

where $A_{i j}=\left(\mathbf{H}_{i j}-E \mathbf{S}_{i j}\right), \mathbf{H}_{i j}=\int \phi_{i} H \phi_{j} \partial \tau$, and $\mathbf{S}_{i j}=\int \phi_{i} \phi_{j} \partial \tau$ are shown below:

\section{2,6-Dithiaadamantane (3)}

$$
\left|\begin{array}{cc}
X & 0 \\
0 & X
\end{array}\right|=0
$$

$X=0,0$

2,4-Dithiaadamantane (4)

$$
\begin{aligned}
& \left|\begin{array}{cc}
X & 1 \\
1 & X
\end{array}\right|=0 \\
& X=-1,1
\end{aligned}
$$

\section{2,4,10-Trithiaadamantane (5)}

$$
\left|\begin{array}{ccc}
X & -1 & -1 \\
-1 & X & -1 \\
-1 & -1 & X
\end{array}\right|=0
$$

$X=-1,-1,2$

2,4,9-Trithiaadamantane (6)

$$
\begin{aligned}
& \left|\begin{array}{ccc}
X & 1 & 1 \\
1 & X & 1 \\
1 & 1 & X
\end{array}\right|=0 \\
& X=-2,1,1
\end{aligned}
$$


2,4,6-Trithiaadamantane (7)

$$
\begin{aligned}
& \left|\begin{array}{ccc}
X & -1 & 0 \\
-1 & X & -1 \\
0 & -1 & X
\end{array}\right|=0 \\
& X=-\sqrt{2}, 0, \sqrt{2}
\end{aligned}
$$

2,4,6,10-Tetrathiaadamantane (8)

$$
\begin{aligned}
& \left|\begin{array}{cccc}
X & -1 & -1 & -1 \\
-1 & X & -1 & 0 \\
-1 & -1 & X & 1 \\
-1 & 0 & 1 & X
\end{array}\right|=0 \\
& X=-2,-1,1,2
\end{aligned}
$$

\section{2,4,6,8-Tetrathiaadamantane (9)}

$$
\begin{aligned}
& \left|\begin{array}{cccc}
X & 1 & 1 & 0 \\
1 & X & 0 & 1 \\
1 & 0 & X & 1 \\
0 & 1 & 1 & X
\end{array}\right|=0 \\
& X=-2,0,0,2
\end{aligned}
$$

2,4,6,8,9-Pentathiaadamantane (10)

$$
\begin{aligned}
& \left|\begin{array}{ccccc}
X & -1 & -1 & 1 & 0 \\
-1 & X & -1 & 0 & 1 \\
-1 & -1 & X & -1 & -1 \\
1 & 0 & -1 & X & -1 \\
0 & 1 & -1 & -1 & X
\end{array}\right|=0 \\
& X=-2,-2,0,2,2
\end{aligned}
$$

2,4,6,8,9,10-Hexathiaadamantane (11)

$$
\left|\begin{array}{cccccc}
X & -1 & -1 & 1 & 0 & 1 \\
-1 & X & -1 & 0 & 1 & -1 \\
-1 & -1 & X & -1 & -1 & 0 \\
1 & 0 & -1 & X & -1 & -1 \\
0 & 1 & -1 & -1 & X & 1 \\
1 & -1 & 0 & -1 & 1 & X
\end{array}\right|=0
$$

$X=-2,-2,-2,2,2,2$ 
Prediction of Hole Mobilities. Hole mobilities for hexathiaadamantane were approximated using the electron coupling matrix element and reorganization energy calculated from DFT calculations. An incoherent hopping model where charge transfer only occurs between neighbors has been used previously for organic semiconductors. ${ }^{3,4}$ If each hopping event is considered a nonadiabatic transfer reaction, standard Marcus theory can be used to express the hopping rate between neighboring molecules, $W$, as a function of reorganization energy $(\lambda)$ and the coupling matrix element $(V){ }^{5,6}$ Assuming temperature to be sufficiently high, vibrational modes can be treated classically. This gives

$$
W=\frac{V^{2}}{\hbar}\left(\frac{\pi}{\lambda k_{B} T}\right)^{1 / 2} \exp \left(-\frac{\lambda}{4 k_{B} T}\right)
$$

where $k_{B}$ is the Boltzmann constant and $T$ is temperature. From the hopping rate, the diffusion coefficient can be evaluated as

$$
D=\frac{1}{2 n} \sum_{i} r^{2} W_{i} P_{i}
$$

for a three-dimensional $(n=3)$ system where $W_{i}$ is the hopping rate due to charge carrier to the $i$ th neighbor, and $r_{i}$ is the distance to neighbor $i$. $P$ is the relative probability for charge carrier to a particular ith neighbor and is evaluated as

$$
P_{i}=W_{i} / \sum_{i} W_{i}
$$

Summing over all possible charge transfer events leads to the diffusion coefficient $D$. The drift mobility of hopping, $\mu$, is then evaluated from the Einstein relation,

$$
\mu=\frac{e}{k_{B} T} D
$$

where $e$ is the electronic charge.

The electron-transfer coupling matrix element and reorganization energy are obtained from DFT calculations on the hexathiaadamantane dimer, and the Marcus-Hush two-state model can be used to approximate the electron-transfer coupling matrix element (V) from orbital energies. ${ }^{5-8}$ For identical isolated molecules,

$$
\mathrm{V}=\frac{1}{2}\left(E_{\text {номо }}-E_{\text {номо-1 }}\right)
$$

where $E_{\text {Номо }}$ is the highest occupied lone-pair orbital localized on a molecule and $E_{\text {Номо- }}$ 1 is the highest-occupied lone-pair orbital localized on a neighboring molecule. The coupling element, $V$, is determined to be $0.14 \mathrm{eV}$ for the hexathiaadamantane dimer.

Reorganization energy, $\lambda$, is taken as the energy due only to relaxation of the molecular geometry associated with charge transfer and does not include reorganization of the solvent. In this case, the reorganization energy is defined as

$$
\lambda=\left(E_{+}^{*}-E_{+}\right)+\left(E^{*}-E\right)
$$


where $E$ and $E_{+}$are the energies of the optimized neutral and cation geometries, respectively, and $E^{*}$ is a single-point energy calculation of the neutral molecule in the optimized cation geometry and $E_{+}^{*}$ is a single-point energy calculation of the cation molecule in the optimized neutral geometry.

Table S3. Reorganization Energy $(\lambda)$ of 2,4,6,8,9,10-Hexathiaadamantane Calculated with B3LYP/6-31G(d)

\begin{tabular}{cccccc}
\hline \multicolumn{7}{c}{ Reorganization Energy $(\lambda)$} \\
$\varepsilon_{0}(\mathrm{E})(\mathrm{au})$ & $\varepsilon_{0}\left(\mathrm{E}^{*}\right)(\mathrm{au})$ & $\varepsilon_{0}\left(\mathrm{E}_{+}\right)(\mathrm{au})$ & $\varepsilon_{0}\left(\mathrm{E}^{*}\right)(\mathrm{au})$ & $\lambda(\mathrm{au})$ & $(\mathrm{eV})$ \\
\hline-2543.921382 & -2543.917336 & -2543.624676 & -2543.620685 & 0.008037 & 0.22 \\
\hline
\end{tabular}

Table S4. Parameters for Calculating Hole Mobility of 2,4,6,8,9,10-Hexathiaadamantane Crystals Calculated with B3LYP/6-31G(d)

\begin{tabular}{cccccccc}
\hline Dimer & $\mathrm{E}_{\text {Hомо }}(\mathrm{eV})$ & $\mathrm{E}_{\text {Hомо-1 }}(\mathrm{eV})$ & $\mathrm{V}(\mathrm{eV})$ & $\mathrm{W}_{\mathrm{i}}\left(\mathrm{s}^{-1}\right)$ & $\mathrm{P}_{\mathrm{i}}$ & $\mathrm{r}(\AA)$ & $\mathrm{r}^{2} \mathrm{~W}_{\mathrm{i}} \mathrm{P}_{\mathrm{i}}\left(\mathrm{cm}^{2} \mathrm{~s}^{-1}\right)$ \\
\hline D1 & -6.17 & -6.43 & 0.13 & $7.40 \mathrm{E}+13$ & $1.15 \mathrm{E}-01$ & 6.354 & $3.44 \mathrm{E}-02$ \\
D1 & -6.17 & -6.43 & 0.13 & $7.40 \mathrm{E}+13$ & $1.15 \mathrm{E}-01$ & 6.354 & $3.44 \mathrm{E}-02$ \\
D1 & -6.17 & -6.43 & 0.13 & $7.40 \mathrm{E}+13$ & $1.15 \mathrm{E}-01$ & 6.354 & $3.44 \mathrm{E}-02$ \\
D1 & -6.17 & -6.43 & 0.13 & $7.40 \mathrm{E}+13$ & $1.15 \mathrm{E}-01$ & 6.354 & $3.44 \mathrm{E}-02$ \\
D1 & -6.17 & -6.43 & 0.13 & $7.40 \mathrm{E}+13$ & $1.15 \mathrm{E}-01$ & 6.354 & $3.44 \mathrm{E}-02$ \\
D1 & -6.17 & -6.43 & 0.13 & $7.40 \mathrm{E}+13$ & $1.15 \mathrm{E}-01$ & 6.354 & $3.44 \mathrm{E}-02$ \\
D1 & -6.17 & -6.43 & 0.13 & $7.40 \mathrm{E}+13$ & $1.15 \mathrm{E}-01$ & 6.354 & $3.44 \mathrm{E}-02$ \\
D1 & -6.17 & -6.43 & 0.13 & $7.40 \mathrm{E}+13$ & $1.15 \mathrm{E}-01$ & 6.354 & $3.44 \mathrm{E}-02$ \\
D2 & -6.21 & -6.32 & 0.05 & $1.25 \mathrm{E}+13$ & $1.94 \mathrm{E}-02$ & 7.084 & $1.22 \mathrm{E}-03$ \\
D2 & -6.21 & -6.32 & 0.05 & $1.25 \mathrm{E}+13$ & $1.94 \mathrm{E}-02$ & 7.084 & $1.22 \mathrm{E}-03$ \\
D2 & -6.21 & -6.32 & 0.05 & $1.25 \mathrm{E}+13$ & $1.94 \mathrm{E}-02$ & 7.084 & $1.22 \mathrm{E}-03$ \\
D2 & -6.21 & -6.32 & 0.05 & $1.25 \mathrm{E}+13$ & $1.94 \mathrm{E}-02$ & 7.084 & $1.22 \mathrm{E}-03$ \\
D3 & -6.31 & -6.31 & 0.00 & $0.00 \mathrm{E}+00$ & $0.00 \mathrm{E}+00$ & 7.819 & $0.00 \mathrm{E}+00$ \\
D3 & -6.31 & -6.31 & 0.00 & $0.00 \mathrm{E}+00$ & $0.00 \mathrm{E}+00$ & 7.819 & $0.00 \mathrm{E}+00$ \\
\hline
\end{tabular}

Table S5. Hole Mobility of 2,4,6,8,9,10-Hexathiaadamantane Crystals Calculated with B3LYP/6-31G(d)

\begin{tabular}{cccc}
\hline \multicolumn{4}{c}{ Hole Mobility $(\mu)$} \\
\hline Compound & $\mathrm{D}\left(\mathrm{cm}^{2} / \mathrm{s}\right)$ & $\mu\left(\mathrm{cm}^{2} /(\mathrm{V} \cdot \mathrm{s})\right)_{\text {calc }}$ & $\mu\left(\mathrm{cm}^{2} /(\mathrm{V} \cdot \mathrm{s})\right)_{\exp }$ \\
\hline Hexathiaadamantane & $4.67 \mathrm{E}-02$ & 1.82 & - \\
\hline
\end{tabular}




\section{References}

(1) Becke, A. D. J. Chem. Phys. 1993, 98, 5648-5652.

(2) Lee, C.; Yang, W.; Parr, R. G. Phys. Rev. B: Condens. Matter Mater. Phys. 1988, 37, 785-789.

(3) Berlin, Y. A.; Hutchison, G. R.; Rempala, P.; Ratner, M. A.; Michl, J. J. Phys. Chem. A 2003, 107, 3970-3980.

(4) Deng, W.-Q.; Goddard, W. A., III J. Phys. Chem. B 2004, 108, 8614-8621.

(5) Marcus, R. A. J. Chem. Phys. 1956, 24, 966-978.

(6) Marcus, R. A.; Eyring, H. Ann. Rev. Phys. Chem. 1964, 15, 155-196.

(7) Hush, N. S. J. Chem. Phys. 1958, 28, 962-972.

(8) Hush, N. S. Trans. Faraday Soc. 1961, 57, 557-980.

\section{Software Citations}

\section{Gaussian 03}

Gaussian 03, Revision C.02, Frisch, M. J.; Trucks, G. W.; Schlegel, H. B.; Scuseria, G. E.; Robb, M. A.; Cheeseman, J. R.; Montgomery, J., J. A.; Vreven, T.; Kudin, K. N.; Burant, J. C.; Millam, J. M.; Iyengar, S. S.; Tomasi, J.; Barone, V.; Mennucci, B.; Cossi, M.; Scalmani, G.; Rega, N.; Petersson, G. A.; Nakatsuji, H.; Hada, M.; Ehara, M.; Toyota, K.; Fukuda, R.; Hasegawa, J.; Ishida, M.; Nakajima, T.; Honda, Y.; Kitao, O.; Nakai, H.; Klene, M.; Li, X.; Knox, J. E.; Hratchian, H. P.; Cross, J. B.; Adamo, C.; Jaramillo, J.; Gomperts, R.; Stratmann, R. E.; Yazyev, O.; Austin, A. J.; Cammi, R.; Pomelli, C.; Ochterski, J. W.; Ayala, P. Y.; Morokuma, K.; Voth, G. A.; Salvador, P.; Dannenberg, J. J.; Zakrzewski, V. G.; Dapprich, S.; Daniels, A. D.; Strain, M. C.; Farkas, O.; Malick, D. K.; Rabuck, A. D.; Raghavachari, K.; Foresman, J. B.; Ortiz, J. V.; Cui, Q.; Baboul, A. G.; Clifford, S.; Cioslowski, J.; Stefanov, B. B.; Liu, G.; Liashenko, A.; Piskorz, P.; Komaromi, I.; Martin, R. L.; Fox, D. J.; Keith, T.; Al-Laham, M. A.; Peng, C. Y.; Nanayakkara, A.; Challacombe, M.; Gill, P. M. W.; Johnson, B.; Chen, W.; Wong, M. W.; Gonzalez, C.; and Pople, J. A., Gaussian, Inc.,Wallingford CT, 2004.

\section{Crystal Structure}

The crystal structure of HTA (11) is provided in the file supporting_info_hta_wudl_cs.cif included with the Supporting Information. 


\section{Geometry Summaries}

Cartesian coordinates and distances are reported in $\AA$. Electronic energies are reported in Hartrees. Charge/multiplicity is shown in parentheses for each structure.

$\mathrm{H}_{2} \mathrm{~S}(0 / 1)$

RB3LYP/6-31G(d) optimized structure:

$\begin{array}{llll}\mathrm{S} & -1.953518 & 2.331315 & 0.000000 \\ \mathrm{H} & -0.618511 & 2.527223 & 0.00000 \odot \\ \mathrm{H} & -2.214480 & 3.655143 & 0.00000 \odot\end{array}$

$E(R B+H F-L Y P)=-399.385435412$

$\operatorname{MeSH}(0 / 1)$

RB3LYP/6-31G(d) optimized structure:

$\begin{array}{lrrr}\text { S } & 0.051205 & -0.821013 & -0.0000443 \\ \text { C } & 1.341387 & 0.484649 & 0.000057 \\ \text { H } & 1.280377 & 1.107026 & 0.895812 \\ \text { H } & 2.302517 & -0.034877 & 0.000033 \\ \text { H } & 1.280397 & 1.107143 & -0.895618 \\ \text { H } & -1.016884 & 0.005878 & -0.000001 \\ & & & \\ \text { E (RB+HF-LYP })= & -438.698348337 & \end{array}$

$\mathrm{Me}_{2} \mathrm{~S}(0 / 1)$

RB3LYP/6-31G(d) optimized structure:

$\begin{array}{lrrr}\text { S } & -1.274087 & 0.268849 & 0.000078 \\ \text { C } & 0.544975 & 0.426965 & 0.000025 \\ \text { H } & 0.955905 & -0.586174 & 0.000069 \\ \text { H } & 0.898563 & 0.949515 & -0.894583 \\ \text { H } & 0.898608 & 0.949611 & 0.894559 \\ \text { C } & -1.732256 & 2.036352 & -0.000022 \\ \text { H } & -1.357690 & 2.544071 & -0.894633 \\ \text { H } & -2.824454 & 2.085520 & -0.000001 \\ \text { H } & -1.357651 & 2.544183 & 0.894509 \\ & & & \\ \text { E(RB+HF-LYP })= & -478.013808483 & \end{array}$

MeSEt (0/1)

RB3LYP/6-31G(d) optimized structure:

$\begin{array}{rrrr}\text { S } & 0.543024 & -0.606930 & -0.000152 \\ \text { C } & -0.860117 & 0.580146 & 0.000039 \\ \text { C } & -2.189075 & -0.174169 & -0.000109 \\ \text { H } & -0.781326 & 1.220115 & -0.886488 \\ \text { H } & -0.781343 & 1.219801 & 0.886784 \\ \text { H } & -3.024896 & 0.534589 & 0.000001 \\ \text { H } & -2.283947 & -0.809958 & -0.886753 \\ \text { H } & -2.283976 & -0.810247 & 0.886332 \\ \text { C } & 1.940083 & 0.568558 & 0.000056 \\ \text { H } & 1.929847 & 1.199828 & 0.894554 \\ \text { H } & 2.859002 & -0.023999 & -0.000031 \\ \text { H } & 1.929867 & 1.200123 & -0.894234 \\ & & & \end{array}$

$\mathrm{Et}_{2} \mathrm{~S}(0 / 1)$

RB3LYP/6-31G(d) optimized structure:

$\begin{array}{rrrr}\text { S } & -1.626432 & -0.551971 & 0.000167 \\ \text { C } & -2.091415 & 1.225973 & -0.000015 \\ \text { C } & -3.613092 & 1.375022 & -0.000010 \\ \text { H } & -1.659950 & 1.705086 & -0.886584 \\ \text { H } & -1.659926 & 1.705275 & 0.886439 \\ \text { H } & -3.887745 & 2.435996 & -0.000105 \\ \text { H } & -4.056485 & 0.909521 & -0.886510 \\ \text { H } & -4.056462 & 0.909687 & 0.886590 \\ \text { C } & 0.204745 & -0.396792 & 0.000105 \\ \text { C } & 0.853191 & -1.781435 & 0.000223 \\ \text { H } & 0.512459 & 0.169984 & 0.886553 \\ \text { H } & 0.512414 & 0.169812 & -0.886470 \\ \text { H } & 1.944990 & -1.686174 & 0.000165 \\ \text { H } & 0.562511 & -2.354652 & 0.886845 \\ \text { H } & 0.562435 & -2.354837 & -0.886255 \\ & & & \\ \text { E(RB+HF-LYP })= & -556.642532742 & \end{array}$

EtSPr $(0 / 1)$

RB3LYP/6-31G(d) optimized structure:

$\begin{array}{rrrr}\text { S } & -1.760700 & -0.511122 & 0.000152 \\ \mathrm{C} & -2.192592 & 1.275345 & -0.000013 \\ \mathrm{C} & -3.711214 & 1.451930 & -0.000043 \\ \mathrm{H} & -1.752242 & 1.746334 & -0.886513 \\ \mathrm{H} & -1.752258 & 1.746494 & 0.886409 \\ \mathrm{H} & -3.966823 & 2.517659 & -0.000127 \\ \mathrm{H} & -4.162970 & 0.994494 & -0.886544 \\ \mathrm{H} & -4.162988 & 0.994629 & 0.886518 \\ \mathrm{C} & 0.072674 & -0.390190 & 0.000156 \\ \mathrm{C} & 0.705901 & -1.785138 & 0.000308 \\ \mathrm{H} & 0.393162 & 0.171058 & 0.886736 \\ \mathrm{H} & 0.393179 & 0.170874 & -0.886535 \\ \mathrm{C} & 2.237828 & -1.723664 & 0.000296 \\ \mathrm{H} & 0.357075 & -2.340697 & 0.879948 \\ \mathrm{H} & 0.357069 & -2.340892 & -0.879208 \\ \mathrm{H} & 2.670685 & -2.730240 & 0.000418 \\ \mathrm{H} & 2.615363 & -1.198417 & -0.885353 \\ \mathrm{H} & 2.615368 & -1.198200 & 0.885815\end{array}$

$E(R B+H F-L Y P)=-595.956301297$

$\operatorname{Pr}_{2} \mathrm{~S}(0 / 1)$

RB3LYP/6-31G(d) optimized structure:

$\begin{array}{rrrr}\text { S } & -1.772558 & -0.553374 & 0.000125 \\ \text { C } & 0.058403 & -0.397785 & 0.000165 \\ \text { C } & 0.716470 & -1.781228 & 0.000296 \\ \text { H } & 0.368103 & 0.169281 & 0.886810 \\ \text { H } & 0.368155 & 0.169136 & -0.886553 \\ \text { C } & 2.246995 & -1.693150 & 0.000304 \\ \text { H } & 0.377221 & -2.342856 & 0.879857 \\ \text { H } & 0.377241 & -2.343011 & -0.879173 \\ \text { H } & 2.697261 & -2.692053 & 0.000405 \\ \text { H } & 2.615500 & -1.161398 & -0.885291\end{array}$




$\begin{array}{lr}\mathrm{H} & 2.615483 \\ \mathrm{C} & -2.237080 \\ \mathrm{C} & -3.760838 \\ \mathrm{H} & -1.805977 \\ \mathrm{H} & -1.806017 \\ \mathrm{C} & -4.188712 \\ \mathrm{H} & -4.177065 \\ \mathrm{H} & -4.177103 \\ \mathrm{H} & -5.280620 \\ \mathrm{H} & -3.810408 \\ \mathrm{H} & -3.810363\end{array}$

-1.161226
1.224502
1.383020
1.705656
1.705802
2.855158
0.875678
0.875798
2.946156
3.380106
3.379991

0.885802

$-\odot .000 \odot 33$

$-0.000078$

$-0.886744$

0.886619

$-0.000186$

$-0.879567$

๑. 879462

$-0.000221$

$\odot .885336$

$-0.885758$

$E(R B+H F-L Y P)=-635.270028538$

$\mathrm{Bu}_{2} \mathrm{~S}(0 / 1)$

RB3LYP/6-31G(d) optimized structure:

S
$\mathrm{C}$
$\mathrm{C}$
$\mathrm{H}$
$\mathrm{H}$
$\mathrm{C}$
$\mathrm{H}$
$\mathrm{H}$
$\mathrm{C}$
$\mathrm{H}$
$\mathrm{H}$
$\mathrm{H}$
$\mathrm{H}$
$\mathrm{H}$
$\mathrm{C}$
$\mathrm{C}$
$\mathrm{H}$
$\mathrm{H}$
$\mathrm{C}$
$\mathrm{H}$
$\mathrm{H}$
$\mathrm{C}$
$\mathrm{H}$
$\mathrm{H}$
$\mathrm{H}$
$\mathrm{H}$
$\mathrm{H}$

-1.584937
-2.049280
-3.572738
-1.617897
-1.617018
-4.011032
-3.991400
-3.990380
-5.534238
-3.586551
-3.587261
-5.818675
-5.984286
-5.983592
0.246192
0.903812
0.554955
0.554932
2.437220
0.564668
0.565805
3.103155
2.773237
2.771816
4.195686
2.812842
2.814037

$-0.484117$

1. 294001

1. 452151

1. 774311

1. 774601

2. 923657

๑. 944114

๑. 945684

3. 090718

3. 430438

3. 428677

4. 149004

2. 623368

2. 624884

$-0.328278$

$-1.711562$

๑. 238694

๑. 239615

$-1.633517$

$-2.275794$

- 2.274312

$-3.013588$

$-1.064218$

$-1.065472$

$-2.928447$

$-3.593825$

$-3.592668$
๑. . 002987

๑. 002830

0.003561

$-0.884088$

$\odot .889162$

0.002355

$-0.875594$

๑. 884085

○. 002820

๑. 880427

$-0.877074$

0.002033

๑. 888246

๑. 003140

$\odot .002411$

๑. 890057

๑. 003615

๑. 881569

$-0.878111$

0.003151

$-0.874460$

0.883042

0.003937

0.887427

$-0.882273$
$-0.881453$

$-0.883193$

$E(R B+H F-L Y P)=-713.897497274$

$\operatorname{PrS}(i-\mathrm{Bu})(0 / 1)$

RB3LYP/6-31G(d) optimized structure:

$\begin{array}{ll}\mathrm{S} & -1.309105 \\ \mathrm{C} & -1.734758 \\ \mathrm{C} & -3.252215 \\ \mathrm{H} & -1.246881 \\ \mathrm{H} & -1.340541 \\ \mathrm{C} & -3.647838 \\ \mathrm{H} & -3.633292 \\ \mathrm{H} & -3.724786 \\ \mathrm{H} & -4.736046 \\ \mathrm{H} & -3.305542 \\ \mathrm{H} & -3.212481 \\ \mathrm{C} & 0.525493 \\ \mathrm{C} & 1.208378 \\ \mathrm{H} & 0.806502 \\ \mathrm{H} & 0.860757 \\ \mathrm{C} & 2.728265 \\ \mathrm{H} & 0.846980\end{array}$

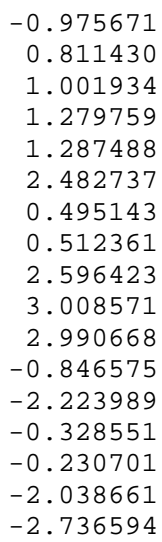

$\odot .035439$

๑. . 005477

$-0.075225$

$-0.858571$

$\odot .911857$

$-0.110676$

$-0.970847$

$\odot .785612$

$-0.169031$

$\odot .788880$

$-0.979724$

0.117952

0.059835

1.044481

$-0.725361$

$-0.077002$

$-0.843032$

$\begin{array}{lrrr}\text { H } & 3.233748 & -3.008508 & -0.151110 \\ H & 2.984905 & -1.457113 & -0.970338 \\ H & 3.141313 & -1.515318 & 0.795072 \\ \mathrm{C} & 0.871612 & -3.096308 & 1.277777 \\ \mathrm{H} & 1.214633 & -2.617966 & 2.204580 \\ \mathrm{H} & -0.205479 & -3.267170 & 1.370596 \\ \mathrm{H} & 1.364731 & -4.072593 & 1.203549 \\ & & & \end{array}$

$(i-\mathrm{Bu})_{2} \mathrm{~S}(0 / 1)$

RB3LYP/6-31G(d) optimized structure:

$\begin{array}{lrrr}\mathrm{S} & 0.021203 & -0.000576 & -0.018225 \\ \mathrm{C} & -0.010385 & -0.000138 & 1.825869 \\ \mathrm{C} & 1.390918 & 0.000032 & 2.465470 \\ \mathrm{C} & 2.189402 & 1.270430 & 2.137963 \\ \mathrm{C} & -1.801837 & -0.000700 & -0.297882 \\ \mathrm{C} & -2.196122 & -0.001286 & -1.786935 \\ \mathrm{C} & -1.739309 & 1.269025 & -2.519318 \\ \mathrm{C} & -1.738968 & -1.272013 & -2.518377 \\ \mathrm{C} & 2.189352 & -1.270609 & 2.138786 \\ \mathrm{H} & -0.565702 & -0.886496 & 2.156889 \\ \mathrm{H} & -0.565709 & 0.886378 & 2.156449 \\ \mathrm{H} & 1.202104 & 0.000383 & 3.550324 \\ \mathrm{H} & 2.430411 & 1.327812 & 1.070735 \\ \mathrm{H} & 1.625630 & 2.173655 & 2.400110 \\ \mathrm{H} & 3.132386 & 1.287676 & 2.696817 \\ \mathrm{H} & -2.221509 & 0.885920 & 0.193404 \\ \mathrm{H} & -2.221481 & -0.886954 & 0.194086 \\ \mathrm{H} & -3.297280 & -0.001424 & -1.783657 \\ \mathrm{H} & -2.131294 & 1.285698 & -3.542989 \\ \mathrm{H} & -2.093095 & 2.172295 & -2.008129 \\ \mathrm{H} & -0.646758 & 1.326862 & -2.577079 \\ \mathrm{H} & -2.130897 & -1.289524 & -3.542056 \\ \mathrm{H} & -0.646398 & -1.329626 & -2.576046 \\ \mathrm{H} & -2.092559 & -2.175001 & -2.006555 \\ \mathrm{H} & 2.430388 & -1.328676 & 1.071603 \\ \mathrm{H} & 3.132318 & -1.287549 & 2.697679 \\ \mathrm{H} & 1.625529 & -2.173642 & 2.401485\end{array}$

$E(R B+H F-L Y P)=-713.894380402$

$(i-\operatorname{Pr})_{2} \mathrm{~S}(0 / 1)$

RB3LYP/6-31G(d) optimized structure:

$\begin{array}{lrrr}\mathrm{S} & -1.679410 & -0.491895 & 0.000095 \\ \mathrm{C} & -2.142401 & 1.241335 & 0.464583 \\ \mathrm{C} & -3.524079 & 1.171874 & 1.125155 \\ \mathrm{C} & -2.135542 & 2.196602 & -0.731614 \\ \mathrm{H} & -1.416863 & 1.585359 & 1.210992 \\ \mathrm{H} & -3.514174 & 0.525852 & 2.008523 \\ \mathrm{H} & -3.841585 & 2.174799 & 1.435041 \\ \mathrm{H} & -4.274301 & 0.783876 & 0.426171 \\ \mathrm{H} & -1.150250 & 2.257319 & -1.205284 \\ \mathrm{H} & -2.856479 & 1.872396 & -1.489976 \\ \mathrm{H} & -2.408642 & 3.209660 & -0.407444 \\ \mathrm{C} & 0.109075 & -0.351538 & -0.464483 \\ \mathrm{C} & 0.503374 & -1.677453 & -1.125300 \\ \mathrm{C} & 1.007703 & -0.027345 & 0.731695 \\ \mathrm{H} & 0.191954 & 0.447256 & -1.210774 \\ \mathrm{H} & -0.109184 & -1.882979 & -2.008654 \\ \mathrm{H} & 1.554770 & -1.643006 & -1.435265 \\ \mathrm{H} & 0.387243 & -2.514152 & -0.426443 \\ \mathrm{H} & 0.737094 & 0.921906 & 1.205547 \\ \mathrm{H} & 0.941981 & -0.815211 & 1.489930 \\ \mathrm{H} & 2.053872 & 0.052333 & 0.407449\end{array}$


$E(R B+H F-L Y P)=-635.271324884$

$(t-\mathrm{Bu})_{2} \mathrm{~S}(0 / 1)$

RB3LYP/6-31G(d) optimized structure:

$\begin{array}{rrrr}\text { S } & -2.168345 & -1.012234 & 0.191059 \\ \text { C } & -2.857953 & 0.733475 & 0.070114 \\ \text { C } & -2.638499 & 1.515754 & 1.375426 \\ \text { C } & -2.325837 & 1.520521 & -1.134393 \\ \text { H } & -4.366357 & 0.477053 & -0.116579 \\ \text { H } & -1.031581 & 0.961692 & 2.233434 \\ \text { H } & -1.581148 & 1.720888 & 1.560393 \\ \text { H } & -2.160913 & 2.481100 & 1.322631 \\ \text { H } & -2.877544 & 0.961590 & -2.065915 \\ \text { H } & -1.266570 & 2.466243 & -1.224374 \\ \text { H } & -4.899896 & 1.773446 & -1.028117 \\ \text { H } & -4.569097 & -0.046544 & -1.056069 \\ \text { H } & -4.776474 & -0.120984 & 0.704718 \\ \text { C } & -0.299205 & -1.072930 & -0.010292 \\ \text { C } & 0.110533 & -0.956387 & -1.487602 \\ \text { C } & 0.443312 & -0.038833 & 0.845690 \\ \text { C } & 0.038530 & -2.488872 & 0.496551 \\ \text { H } & -0.407530 & -1.703672 & -2.096577 \\ \text { H } & -0.112273 & 0.030558 & -1.900639 \\ \text { H } & 1.192105 & -1.123055 & -1.587720 \\ \text { H } & 0.141309 & -0.099254 & 1.895736 \\ \text { H } & 1.523947 & -0.228924 & 0.791122 \\ \text { H } & 0.277288 & 0.984318 & 0.495122 \\ \text { H } & 1.112004 & -2.676508 & 0.365867 \\ \text { H } & -0.204414 & -2.601623 & 1.557758 \\ \text { H } & -0.506965 & -3.256395 & -0.063553\end{array}$

$E(R B+H F-L Y P)=-713.891031218$

1 - Adamantane $(0 / 1)$

RB3LYP/6-311+G(d,p) optimized structure:

$\begin{array}{rrrr}\text { C } & 1.640882 & 0.688800 & -0.055373 \\ \text { H } & 2.031444 & 1.497883 & 0.573409 \\ \text { H } & 2.455167 & 0.385520 & -0.724290 \\ \text { C } & 0.438282 & 1.192231 & -0.879948 \\ \text { H } & 0.748399 & 2.037208 & -1.504650 \\ \text { C } & 1.206529 & -0.501863 & 0.823786 \\ \text { H } & 2.062173 & -0.857634 & 1.408467 \\ \text { C } & 0.081101 & -0.049459 & 1.776845 \\ \text { H } & 0.444269 & 0.746928 & 2.437590 \\ \text { H } & -0.223124 & -0.882443 & 2.421829 \\ \text { C } & -0.685721 & 1.641028 & 0.076495 \\ \text { H } & -1.539973 & 2.018537 & -0.498194 \\ \text { H } & -0.336801 & 2.467548 & 0.707002 \\ \text { C } & -1.124751 & 0.452529 & 0.956480 \\ \text { H } & -1.922757 & 0.773308 & 1.635420 \\ \text { C } & -1.640577 & -0.688682 & 0.055949 \\ \text { H } & -1.973782 & -1.532786 & 0.671402 \\ \text { H } & -2.511338 & -0.350788 & -0.518553 \\ \text { C } & -0.081543 & 0.049852 & -1.776931 \\ \text { H } & 0.702056 & -0.265150 & -2.476386 \\ \text { H } & -0.925476 & 0.401300 & -2.382429 \\ \text { C } & 0.686196 & -1.640569 & -0.077458 \\ \text { H } & 1.483617 & -1.984382 & -0.747054 \\ \text { H } & 0.393242 & -2.500703 & 0.536294 \\ \text { C } & -0.519126 & -1.142370 & -0.901329 \\ \text { H } & -0.886732 & -1.952247 & -1.541272\end{array}$

$E(R B+H F-L Y P)=-390.823793811$
2 - 2-Thiaadamantane $(0 / 1)$

RB3LYP/6-311+G(d,p) optimized structure:

$\begin{array}{lrrr}\text { C } & -1.313534 & 0.237718 & -0.001027 \\ \text { H } & -0.217707 & 0.247172 & 0.016425 \\ \text { H } & -1.648016 & 1.285169 & -0.043546 \\ \text { C } & -1.852979 & -0.402477 & 1.291771 \\ \text { H } & -1.508337 & 0.156618 & 2.168070 \\ \text { C } & -1.831821 & -0.508559 & -1.250518 \\ \text { H } & -1.457686 & 0.004023 & -2.146532 \\ \text { C } & -1.341402 & -1.973323 & -1.277529 \\ \text { H } & -0.246205 & -2.013876 & -1.288948 \\ \text { H } & -1.695140 & -2.453747 & -2.202138 \\ \text { C } & -1.882726 & -2.762570 & -0.070784 \\ \text { H } & -1.558300 & -3.807341 & -0.120448 \\ \text { C } & -3.419440 & -2.678851 & -0.010112 \\ \text { H } & -3.836487 & -3.180769 & -0.896108 \\ \text { H } & -3.787862 & -3.216326 & 0.871147 \\ \text { C } & -3.391571 & -0.467809 & 1.266390 \\ \text { H } & -3.789361 & 0.558147 & 1.262484 \\ \text { H } & -3.759364 & -0.955278 & 2.176519 \\ \text { C } & -3.374379 & -0.488940 & -1.250824 \\ \text { H } & -3.739635 & 0.547244 & -1.272884 \\ \text { H } & -3.758895 & -0.980868 & -2.155109 \\ \text { C } & -3.896972 & -1.209711 & 0.009040 \\ \text { H } & -4.994876 & -1.196910 & 0.010837 \\ \text { S } & -1.149030 & -2.104316 & 1.498601\end{array}$

$E(R B+H F-L Y P)=-749.713658186$

3 - 2,6-dithiaadamantane (0/1)

RB3LYP/6-311+G(d,p) optimized structure:

$\begin{array}{rrrr}\text { C } & -0.145920 & -0.509788 & 1.357268 \\ \text { H } & 0.195929 & -0.040545 & 2.285807 \\ \text { C } & -0.147418 & -0.509099 & -1.357008 \\ \text { H } & 0.192782 & -0.038935 & -2.285687 \\ \text { C } & 0.393466 & -1.950311 & -1.283465 \\ \text { H } & 1.489044 & -1.920159 & -1.324930 \\ \text { H } & 0.039928 & -2.514083 & -2.155572 \\ \text { C } & 0.392081 & -1.952343 & 1.283854 \\ \text { H } & 0.033121 & -2.517189 & 2.152770 \\ \text { H } & 1.487872 & -1.927601 & 1.328916 \\ \text { C } & -0.047072 & -2.682203 & -0.000476 \\ \text { H } & 0.363342 & -3.697484 & -0.001345 \\ \text { C } & -1.683791 & -0.441753 & 1.283926 \\ \text { H } & -1.994349 & 0.609325 & 1.325232 \\ \text { H } & -2.110265 & -0.952314 & 2.156193 \\ \text { C } & -1.685653 & -0.444193 & -1.283390 \\ \text { H } & -2.001431 & 0.605398 & -1.328609 \\ \text { H } & -2.111715 & -0.960574 & -2.152148 \\ \text { C } & -2.243487 & -1.087510 & 0.001140 \\ \text { H } & -3.335951 & -1.011636 & 0.002147 \\ \text { S } & 0.593524 & 0.510044 & -0.000021 \\ \text { S } & -1.885471 & -2.904344 & 0.000481\end{array}$

$E(R B+H F-L Y P)=-1108.60271079$

4 - 2,4-Dithiaadamantane (0/1)

$R B 3 L Y P / 6-311+G(d, p)$ optimized structure:

$\begin{array}{rrrr}\mathrm{C} & -1.299240 & 0.239430 & 0.001289 \\ \mathrm{H} & -0.202115 & 0.243115 & -0.022570 \\ \mathrm{H} & -1.635407 & 1.283793 & -0.022441 \\ \mathrm{C} & -1.757187 & -0.391451 & 1.327678\end{array}$




$\begin{array}{lrrr}\text { H } & -1.354930 & 0.162456 & 2.179595 \\ \mathrm{C} & -1.840244 & -0.505523 & -1.240183 \\ \mathrm{H} & -1.464083 & 0.012662 & -2.131622 \\ \mathrm{C} & -1.349418 & -1.968825 & -1.275039 \\ \mathrm{H} & -0.254744 & -2.011918 & -1.292403 \\ \mathrm{H} & -1.709404 & -2.447243 & -2.198156 \\ \mathrm{C} & -1.886474 & -2.752604 & -0.065132 \\ \mathrm{H} & -1.564456 & -3.797934 & -0.114369 \\ \mathrm{C} & -3.425944 & -2.689607 & -0.013258 \\ \mathrm{H} & -3.813825 & -3.223712 & -0.894668 \\ \mathrm{H} & -3.800259 & -3.205250 & 0.875808 \\ \mathrm{C} & -3.383613 & -0.491844 & -1.274855 \\ \mathrm{H} & -3.763501 & 0.535706 & -1.292085 \\ \mathrm{H} & -3.727185 & -0.982182 & -2.197973 \\ \mathrm{C} & -3.962469 & -1.245273 & -0.064944 \\ \mathrm{H} & -5.056142 & -1.262705 & -0.114052 \\ \mathrm{~S} & -1.115709 & -2.114030 & 1.495981 \\ \mathrm{~S} & -3.593711 & -0.314812 & 1.496206 \\ & & & \\ \mathrm{E}(\mathrm{RB}+\mathrm{HF}-\mathrm{LYP})= & -1108.60057900 & \end{array}$

5 - 2,4,10-Trithiaadamantane (0/1)

RB3LYP/6-311+G(d,p) optimized structure:

$\begin{array}{lrrr}\mathrm{C} & -1.770111 & -0.408635 & 1.425050 \\ \mathrm{H} & -1.399401 & 0.101545 & 2.316367 \\ \mathrm{C} & -1.857180 & -0.529335 & -1.342078 \\ \mathrm{H} & -1.512259 & -0.053888 & -2.266059 \\ \mathrm{C} & -1.345736 & -1.980857 & -1.288720 \\ \mathrm{H} & -0.252458 & -2.003023 & -1.303565 \\ \mathrm{H} & -1.700617 & -2.498690 & -2.193425 \\ \mathrm{C} & -1.885057 & -2.743066 & -0.064058 \\ \mathrm{H} & -1.558822 & -3.787198 & -0.110608 \\ \mathrm{C} & -3.423494 & -2.686046 & -0.021157 \\ \mathrm{H} & -3.806993 & -3.213828 & -0.908241 \\ \mathrm{H} & -3.801692 & -3.207917 & 0.862322 \\ \mathrm{C} & -3.395976 & -0.492212 & -1.288184 \\ \mathrm{H} & -3.755558 & 0.540502 & -1.303186 \\ \mathrm{H} & -3.778983 & -0.989957 & -2.192740 \\ \mathrm{C} & -3.954135 & -1.241054 & -0.063657 \\ \mathrm{H} & -5.047921 & -1.254211 & -0.110081 \\ \mathrm{~S} & -1.129105 & -2.133019 & 1.515211 \\ \mathrm{~S} & -3.608031 & -0.333217 & 1.515697 \\ \mathrm{~S} & -1.095872 & 0.519328 & -0.016081\end{array}$

$E(R B+H F-L Y P)=-1467.48334443$

6 - 2,4,9-Trithiaadamantane (0/1)

$R B 3 L Y P / 6-311+G(d, p)$ optimized structure:

$\begin{array}{lrrr}\text { C } & 0.696501 & -1.434470 & -\odot .180889 \\ \text { H } & 1.153998 & -2.376640 & -0.481822 \\ \text { C } & 0.894323 & 1.320179 & -0.183461 \\ \text { H } & 1.481684 & 2.186973 & -0.485558 \\ \text { C } & -1.590481 & 0.114295 & -0.184323 \\ \text { H } & -2.634724 & 0.188959 & -0.486924 \\ \text { C } & -1.465737 & 0.106319 & 1.345763 \\ \text { H } & -1.913150 & 1.025091 & 1.738265 \\ \text { H } & -2.039991 & -0.738557 & 1.739168 \\ \text { C } & 0.821901 & 1.217235 & 1.346503 \\ \text { H } & 1.840718 & 1.146073 & 1.740716 \\ \text { H } & 0.376292 & 2.137233 & 1.738141 \\ \text { C } & -0.001351 & 0.001349 & 1.816433 \\ \text { H } & -0.002068 & 0.002262 & 2.911050 \\ \text { S } & -0.998331 & -1.476002 & -0.909472 \\ \text { S } & 1.777523 & -0.128126 & -0.909506 \\ \text { C } & 0.640838 & -1.320171 & 1.348601 \\ \text { H } & 0.069147 & -2.166847 & 1.742027\end{array}$

\author{
$\begin{array}{llll}\text { H } & 1.659633 & -1.394139 & 1.742179\end{array}$ \\ $\begin{array}{llll}\mathrm{S} & -0.777042 & 1.601538 & -0.914059\end{array}$ \\ $E(R B+H F-L Y P)=-1467.48545291$
}

7 - 2,4,6-Trithiaadamantane (0/1)

RB3LYP/6-311+G(d,p) optimized structure:

$\begin{array}{lrrr}\text { C } & 0.978867 & 0.758152 & 0.968784 \\ \text { H } & 1.612247 & 1.359265 & 1.620718 \\ \text { C } & 0.796405 & -1.125686 & -0.994765 \\ \text { H } & 1.340870 & -1.773875 & -1.684118 \\ \text { C } & 0.000003 & -1.985797 & 0.000042 \\ \text { H } & 0.694417 & -2.631366 & 0.547301 \\ \text { H } & -0.694411 & -2.631386 & -0.547193 \\ \text { C } & -0.796395 & -1.125654 & 0.994819 \\ \text { H } & -1.340859 & -1.773813 & 1.684199 \\ \text { C } & -0.096869 & -0.175943 & -1.815776 \\ \text { H } & 0.529045 & 0.427261 & -2.478292 \\ \text { H } & -0.762250 & -0.783553 & -2.439775 \\ \text { C } & -0.978908 & 0.758070 & -0.968824 \\ \text { H } & -1.612273 & 1.359168 & -1.620785 \\ \text { S } & -0.000008 & 1.984346 & -0.000049 \\ \text { S } & 2.130091 & -0.204647 & -0.098251 \\ \text { C } & 0.096867 & -0.175860 & 1.815783 \\ \text { H } & 0.762275 & -0.783427 & 2.439792 \\ \text { H } & -0.529058 & 0.427349 & 2.478285 \\ \text { S } & -2.130091 & -0.204654 & 0.098263 \\ \text { E(RB+HF-LYP) }= & -1467.48598942 & \end{array}$

8 - 2,4,6,10-Tetrathiaadamantane (0/1)
RB3LYP/6-311+G(d,p) optimized structure:

$\begin{array}{lrrr}\text { C } & -1.773870 & -0.414311 & 1.414958 \\ \text { H } & -1.400698 & 0.095368 & 2.305608 \\ \text { C } & -1.867032 & -0.435071 & -1.376466 \\ \text { H } & -1.561231 & 0.091808 & -2.283847 \\ \text { C } & -1.896703 & -2.819614 & 0.000536 \\ \text { H } & -1.610582 & -3.874393 & 0.006513 \\ \text { C } & -3.430314 & -2.696724 & -0.006297 \\ \text { H } & -3.803644 & -3.235234 & -0.887288 \\ \text { H } & -3.834624 & -3.186034 & 0.886005 \\ \text { C } & -3.402654 & -0.473776 & -1.289983 \\ \text { H } & -3.788125 & 0.551008 & -1.272026 \\ \text { H } & -3.775341 & -0.960684 & -2.200773 \\ \text { C } & -3.941077 & -1.244803 & -0.069894 \\ \text { H } & -5.034294 & -1.262960 & -0.124893 \\ \text { S } & -1.128035 & -2.137080 & 1.535724 \\ \text { S } & -3.608310 & -0.336896 & 1.509492 \\ \text { S } & -1.094737 & 0.539038 & -0.009652 \\ \text { S } & -1.172556 & -2.136204 & -1.553872 \\ & & & \\ \text { E(RB+HF-LYP })= & -1826.36685369 & \end{array}$

9 - 2,4,6,8-Tetrathiaadamantane (0/1) RB3LYP/6-311+G(d,p) optimized structure:

$\begin{array}{rrrr}\text { C } & 1.105092 & -0.700996 & 1.010675 \\ \text { H } & 1.817715 & -1.151729 & 1.700919 \\ \text { C } & -0.703008 & -1.103525 & -1.009046 \\ \text { H } & -1.156317 & -1.815522 & -1.698244 \\ \text { C } & -1.102201 & 0.705208 & 1.010742 \\ \text { H } & -1.812047 & 1.160227 & 1.701036 \\ \text { C } & -0.000640 & -0.000621 & -1.823185 \\ \text { H } & 0.746697 & -0.477384 & -2.464124\end{array}$




$$
\begin{aligned}
& \mathrm{H} \\
& \mathrm{C} \\
& \mathrm{H} \\
& \mathrm{S} \\
& \mathrm{S} \\
& \mathrm{S} \\
& \mathrm{C} \\
& \mathrm{H} \\
& \mathrm{H} \\
& \mathrm{S}
\end{aligned}
$$

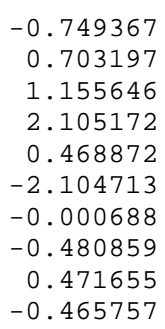

0.475246

1.103724

1. 814204

0.468943

$-2.104045$

$-0.465636$

$-0.001285$

$-0.755426$

○. 740514

2. 105813
$-2.463174$

$-1.012331$

$-0.000615$

0.000440

$\odot .002170$

1. 823192

2.453626

2. 473679

$-0.002016$
$-1.703668$

$E(R B+H F-L Y P)=-1826.36760160$

10 - 2,4,6,8,9-Pentathiaadamantane (0/1)

$R B 3 L Y P / 6-311+G(d, p)$ optimized structure:

$\begin{array}{lrrr}\text { C } & -1.778442 & -0.420652 & 1.413147 \\ \text { H } & -1.404225 & 0.094666 & 2.300378 \\ \text { C } & -1.778801 & -0.420835 & -1.412943 \\ \text { H } & -1.404811 & 0.094370 & -2.300335 \\ \text { C } & -1.897502 & -2.806949 & 0.000271 \\ \text { H } & -1.619158 & -3.863966 & 0.000304 \\ \text { C } & -3.434152 & -2.700956 & 0.000459 \\ \text { H } & -3.812018 & -3.221302 & -0.887356 \\ \text { H } & -3.811792 & -3.221188 & 0.888438 \\ \text { C } & -4.010742 & -1.272644 & 0.000440 \\ \text { H } & -5.101997 & -1.335272 & 0.000584 \\ \text { S } & -1.134450 & -2.140821 & 1.541321 \\ \text { S } & -3.613460 & -0.340950 & 1.541519 \\ \text { S } & -1.081096 & 0.539978 & -0.000049 \\ \text { S } & -1.134839 & -2.141019 & -1.541058 \\ \text { S } & -3.613852 & -0.341152 & -1.540860 \\ & & & \\ \text { E(RB+HF-LYP }= & -2185.24612595 & \end{array}$

11 - 2,4,6,8,9,10-Hexathiaadamantane (0/1)

$R B 3 L Y P / 6-311+G(d, p)$ optimized structure:

$\begin{array}{lrrr}\mathrm{C} & -0.277393 & -1.171742 & 1.403234 \\ \mathrm{H} & 0.093225 & -0.661376 & 2.295215 \\ \mathrm{C} & -0.277786 & -1.171668 & -1.402885 \\ \mathrm{H} & 0.092686 & -0.661251 & -2.294936 \\ \mathrm{C} & -0.307863 & -3.601915 & 0.000128 \\ \mathrm{H} & 0.043423 & -4.636349 & 0.000099 \\ \mathrm{C} & -2.578773 & -1.953208 & 0.000424 \\ \mathrm{H} & -3.671134 & -1.939726 & 0.000526 \\ \mathrm{~S} & -2.110991 & -1.065712 & 1.545347 \\ \mathrm{~S} & 0.424245 & -0.206177 & 0.000049 \\ \mathrm{~S} & -2.111393 & -1.067004 & -1.545282 \\ \mathrm{~S} & 0.389778 & -2.882561 & -1.545669 \\ \mathrm{~S} & 0.390208 & -2.882728 & 1.545753 \\ \mathrm{~S} & -2.144581 & -3.743469 & 0.001174 \\ & & & \end{array}$

$E(R B+H F-L Y P)=-2544.12305163$

$2_{\text {H2S }}(0 / 1)$

RB3LYP/6-311+G(d,p) optimized structure:

$\begin{array}{crrr}\mathrm{S} & 0.829203 & -1.261998 & -0.047004 \\ \mathrm{H} & 2.161765 & -1.062518 & -0.047004 \\ \mathrm{H} & 0.572423 & 0.060717 & -0.047004 \\ & \\ \text { E }(\mathrm{RB}+\mathrm{HF}-\mathrm{LYP})= & -399.422527894 & \end{array}$

$3_{\text {H2S }}(0 / 1)$
RB3LYP/6-311+G(d,p) optimized structure:

$\begin{array}{lrrr}\text { H } & -1.218447 & 0.839612 & 0.465623 \\ \mathrm{~S} & -2.109707 & 0.000000 & -0.000001 \\ \mathrm{H} & -1.218446 & -0.839613 & -0.465624 \\ \mathrm{H} & 1.218447 & -0.465624 & 0.839611 \\ \mathrm{~S} & 2.109708 & 0.000000 & -0.000001 \\ \mathrm{H} & 1.218446 & 0.465624 & -0.839612 \\ & & & \end{array}$

$\mathbf{4}_{\text {H2S }}(0 / 1)$

RB3LYP/6-311+G(d,p) optimized structure:

$\begin{array}{rrrr}\mathrm{H} & -1.350912 & 0.002680 & -0.695530 \\ \mathrm{~S} & -1.531150 & -1.221532 & -0.166966 \\ \mathrm{H} & -0.484497 & -1.210318 & 0.537615 \\ \mathrm{H} & 0.484498 & -1.210317 & 0.537616 \\ \mathrm{~S} & 1.531151 & -1.221531 & -0.166965 \\ \mathrm{H} & 1.350913 & 0.002681 & -0.695530\end{array}$

$E(R B+H F-L Y P)=-798.769641155$

$5_{\text {H2S }}(0 / 1)$

RB3LYP/6-311+G(d,p) optimized structure:

$\begin{array}{lrrr}\text { H } & 0.686035 & 1.403781 & 0.359224 \\ \mathrm{~S} & 0.759786 & 1.579965 & -0.970609 \\ \mathrm{H} & 0.246018 & 0.530036 & -1.290567 \\ \mathrm{H} & 0.320473 & -0.514864 & -1.280407 \\ \mathrm{~S} & 0.977455 & -1.474973 & -0.940912 \\ \mathrm{H} & 0.877704 & -1.285072 & 0.385324 \\ \mathrm{H} & -0.621668 & -0.056774 & -1.272011 \\ \mathrm{~S} & -1.777515 & -0.135701 & -0.916220 \\ \mathrm{H} & -1.547290 & -0.106209 & 0.407095 \\ & & & \end{array}$

$\mathbf{6}_{\mathrm{H} 2 \mathrm{~S}}(0 / 1)$

RB3LYP/6-311+G(d,p) optimized structure:

$\begin{array}{lrrr}\text { S } & -1.777191 & -0.096038 & -0.901164 \\ \text { S } & 0.975693 & -1.490227 & -0.898743 \\ \text { S } & 0.806764 & 1.590197 & -0.895590 \\ \text { H } & -1.054388 & -1.002737 & -0.403756 \\ \text { H } & -0.183634 & -1.443637 & -0.403073 \\ \text { H } & -1.157717 & 0.882924 & -0.401442 \\ \text { H } & -0.340270 & 1.416320 & -0.399725 \\ \text { H } & 1.397484 & -0.411545 & -0.398562 \\ \text { H } & 1.343910 & 0.563006 & -0.397699 \\ \text { E(RB+HF-LYP })= & -1198.05153870 & \end{array}$

$7_{\text {H2S }}(0 / 1)$

RB3LYP/6-311+G(d,p) optimized structure:

$\begin{array}{rrrr}\text { S } & 0.000001 & -1.987175 & 0.000004 \\ \mathrm{~S} & 2.131133 & 0.201821 & 0.072251 \\ \mathrm{~S} & -2.131129 & 0.201820 & -0.072256 \\ \mathrm{H} & -0.660727 & -1.149313 & 0.670072 \\ \mathrm{H} & -1.334415 & -0.457072 & 0.648364 \\ \mathrm{H} & -1.169586 & 0.871387 & -0.735804 \\ \mathrm{H} & 1.169590 & 0.871385 & 0.735802 \\ \mathrm{H} & 0.660720 & -1.149318 & -0.670081 \\ \mathrm{H} & 1.334411 & -0.457082 & -0.648347\end{array}$


$E(R B+H F-L Y P)=-1198.11377484$

$\mathbf{8}_{\mathrm{H} 2 \mathrm{~S}}(0 / 1)$

RB3LYP/6-311+G(d,p) optimized structure:

$\begin{array}{lr}\mathrm{H} & 0.593209 \\ \mathrm{~S} & -0.165055 \\ \mathrm{H} & -0.808915 \\ \mathrm{H} & -1.475737 \\ \mathrm{~S} & -2.116361 \\ \mathrm{H} & -1.062855 \\ \mathrm{H} & -0.808916 \\ \mathrm{~S} & -0.165056 \\ \mathrm{H} & 0.593209 \\ \mathrm{H} & 1.330798 \\ \mathrm{~S} & 2.165110 \\ \mathrm{H} & 1.330798\end{array}$

$$
\begin{array}{r}
1.430219 \\
1.545227 \\
0.529919 \\
0.00000 \odot \\
0.00000 \odot \\
-0.000001 \\
-0.529917 \\
-1.545226 \\
-1.430219 \\
-0.941472 \\
0.0000 \odot \odot \\
0.941472
\end{array}
$$

$-0.284710$

$-1.287054$

$-1.137696$

$-0.528227$

0.501182

1.338784

$-1.137696$

$-1.287055$

$-0.284713$

$\odot .132213$

0.028311

$\odot .132215$

$E(R B+H F-L Y P)=-1597.34453998$

$9_{\mathrm{H} 2 \mathrm{~S}}(0 / 1)$

RB3LYP/6-311+G(d,p) optimized structure:

S
S
S
S
H
H
H
H
H
H
H
H

$$
\begin{array}{r}
1.769378 \\
1.232244 \\
-1.770648 \\
-1.230470 \\
-1.440890 \\
-1.269741 \\
0.718892 \\
-0.231439 \\
1.440388 \\
1.270274 \\
0.232256 \\
-0.719130
\end{array}
$$

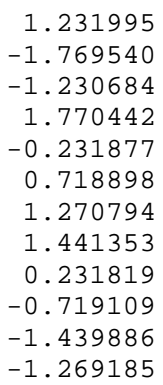

$\odot .002046$

$-0.002138$

$-0.000488$

0.000565

$\odot .690316$

0.690729

$-0.689409$

$-0.689957$

$\odot .691288$

0.689698

$-0.691620$

$-0.69100 \odot$

$E(R B+H F-L Y P)=-1597.37716673$

$10_{\mathrm{H} 2 \mathrm{~S}}(0 / 1)$

RB3LYP/6-311+G(d,p) optimized structure:

$\mathrm{H}$
$\mathrm{S}$
$\mathrm{H}$
$\mathrm{H}$
$\mathrm{S}$
$\mathrm{H}$
$\mathrm{H}$
$\mathrm{S}$
$\mathrm{H}$
$\mathrm{H}$
$\mathrm{S}$
$\mathrm{H}$
$\mathrm{H}$
$\mathrm{S}$
$\mathrm{H}$

$$
\begin{array}{r}
0.441942 \\
1.531754 \\
1.377238 \\
1.377236 \\
1.531747 \\
0.523115 \\
-0.523126 \\
-1.531752 \\
-1.377233 \\
-1.377231 \\
-1.531745 \\
-0.441936 \\
-0.000002 \\
-0.000006 \\
-0.000007
\end{array}
$$

0.545866

$-0.175370$

$-0.849587$

$-0.848957$

$-0.173381$

$\odot .494019$

0.494017

$-0.173390$

$-0.848964$

$-0.849591$

$-0.175375$

○. 545866

1. 183144

2. 026488

1. 247678

$E(R B+H F-L Y P)=-1996.53465610$

$1_{\text {H2S }}(0 / 1)$

RB3LYP/6-311+G(d,p) optimized structure:

$\begin{array}{rrrr}H & -1.205859 & 0.044869 & 1.165068 \\ \mathrm{~S} & -2.128530 & -0.242915 & 0.433803\end{array}$

$\begin{array}{rrrr}\mathrm{H} & -1.521586 & -0.356586 & -0.609171 \\ \mathrm{H} & -0.804487 & -0.900962 & -1.163746 \\ \mathrm{~S} & -0.031873 & -1.834544 & -1.187702 \\ \mathrm{H} & 0.763511 & -1.449930 & -0.357957 \\ \mathrm{H} & 1.502445 & -0.739044 & -0.099948 \\ \mathrm{~S} & 2.128553 & 0.243766 & -0.433195 \\ \mathrm{H} & 1.225016 & 1.051402 & -0.455655 \\ \mathrm{H} & -.327173 & 1.365603 & -0.917391 \\ \mathrm{~S} & -0.496445 & 1.162410 & -1.783136 \\ \mathrm{H} & -0.963397 & 0.124043 & -1.367388 \\ \mathrm{H} & 0.508080 & 1.595539 & 0.098689 \\ \mathrm{~S} & 0.032416 & 1.834678 & 1.187596 \\ \mathrm{H} & -0.466634 & 0.755573 & 1.422934 \\ \mathrm{H} & -0.308096 & -0.270024 & 1.626497 \\ \mathrm{~S} & 0.495850 & -1.163432 & 1.782670 \\ \mathrm{H} & 0.943973 & -1.220406 & 0.657967\end{array}$

$E(R B+H F-L Y P)=-2395.75091202$

D1 (0/1)

RB3LYP/6-31G(d) optimized structure:

$\begin{array}{lllr}\text { S } & 9.085044 & 8.005143 & 7.834901 \\ \text { S } & 7.093636 & 7.099030 & 10.016510 \\ \mathrm{C} & 8.409631 & 6.605927 & 8.824832 \\ \mathrm{H} & 9.243700 & 6.294275 & 9.457678 \\ \mathrm{C} & 5.777420 & 7.589484 & 8.822809 \\ \mathrm{~S} & 8.002005 & 5.108617 & 7.831085 \\ \mathrm{C} & 7.587556 & 8.407824 & 6.839270 \\ \mathrm{~S} & 5.104253 & 6.177293 & 7.844563 \\ \mathrm{H} & 4.944579 & 7.904668 & 9.455500 \\ \mathrm{~S} & 6.188349 & 9.086147 & 7.830661 \\ \mathrm{C} & 6.600636 & 5.775051 & 6.848437 \\ \mathrm{~S} & 7.079078 & 7.091816 & 5.658696 \\ \mathrm{H} & 6.280870 & 4.943691 & 6.216129 \\ \mathrm{H} & 7.898478 & 9.240031 & 6.203734 \\ \mathrm{~S} & 1.547088 & 4.442155 & 3.903362 \\ \mathrm{~S} & 3.525377 & 3.521165 & 1.707182 \\ \mathrm{C} & 2.213763 & 3.041973 & 2.908138 \\ \mathrm{H} & 1.373543 & 2.732496 & 2.282323 \\ \mathrm{C} & 4.841590 & 4.017901 & 2.892174 \\ \mathrm{~S} & 2.624444 & 1.542182 & 3.895648 \\ \mathrm{C} & 3.047876 & 4.848938 & 4.889145 \\ \mathrm{~S} & 5.524963 & 2.629367 & 3.898737 \\ \mathrm{H} & 5.675609 & 4.327907 & 2.258374 \\ \mathrm{~S} & 4.439983 & 5.516057 & 3.884693 \\ \mathrm{C} & 4.022908 & 2.213841 & 4.883410 \\ \mathrm{~S} & 3.536643 & 3.528157 & 6.080736 \\ \mathrm{H} & 4.335751 & 1.377577 & 5.512926 \\ \mathrm{H} & 2.750827 & 5.686885 & 5.523648\end{array}$

$E(R B+H F-L Y P)=-5087.84553742$

D2 (0/1)

RB3LYP/6-31G(d) optimized structure:

$\begin{array}{lllr}\text { S } & 9.036374 & 8.017133 & 7.840387 \\ \text { S } & 7.037381 & 7.093445 & 10.006493 \\ \text { C } & 8.368608 & 6.611735 & 8.827974 \\ \text { H } & 9.200570 & 6.309467 & 9.468053 \\ \text { C } & 5.730913 & 7.570387 & 8.798394 \\ \text { S } & 7.992777 & 5.102474 & 7.837595 \\ \text { C } & 7.548182 & 8.407223 & 6.830525 \\ \text { S } & 5.083527 & 6.161200 & 7.806217 \\ \text { H } & 4.886278 & 7.876121 & 9.420066 \\ \text { S } & 6.129070 & 9.067818 & 7.801090 \\ \text { C } & 6.597268 & 5.764683 & 6.836496 \\ \text { S } & 7.101494 & 7.072162 & 5.644297\end{array}$




$\begin{array}{rrrr}\text { H } & 6.297348 & 4.926589 & 6.202910 \\ \text { H } & 7.859114 & 9.241549 & 6.197754 \\ \text { S } & 16.168723 & 8.008081 & 7.815370 \\ \text { S } & 14.198188 & 7.075338 & 10.001278 \\ \text { C } & 15.510771 & 6.596646 & 8.799626 \\ \text { H } & 16.350994 & 6.288922 & 9.426267 \\ \mathrm{C} & 12.873240 & 7.556047 & 8.812426 \\ \text { S } & 15.116933 & 5.098517 & 7.800268 \\ \text { C } & 14.663359 & 8.404286 & 6.829585 \\ \text { S } & 12.216499 & 6.143697 & 7.828708 \\ \text { H } & 12.037725 & 7.861413 & 9.446398 \\ \text { S } & 13.259726 & 9.059794 & 7.826265 \\ \text { C } & 13.701804 & 5.762353 & 6.818535 \\ \text { S } & 14.185066 & 7.089705 & 5.634709 \\ \text { H } & 13.394478 & 4.929220 & 6.182534 \\ \text { H } & 14.964790 & 9.244794 & 6.200181\end{array}$

$E(R B+H F-L Y P)=-5087.84061913$

D3 (0/1)

RB3LYP/6-31G(d) optimized structure:

$\begin{array}{lrrr}\text { S } & 9.066655 & 8.002705 & 7.773772 \\ \text { S } & 7.083600 & 7.083600 & 9.960100 \\ \text { C } & 8.400604 & 6.600614 & 8.765596 \\ \text { H } & 9.237588 & 6.294240 & 9.396615 \\ \text { C } & 5.766596 & 7.566586 & 8.765596 \\ \text { S } & 8.003056 & 5.100682 & 7.773407 \\ \text { C } & 7.566616 & 8.400730 & 6.781816 \\ \text { S } & 5.100545 & 6.164495 & 7.773772 \\ \text { H } & 4.929612 & 7.872960 & 9.396615 \\ \text { S } & 6.164144 & 9.066518 & 7.773407 \\ \text { C } & 6.600584 & 5.766470 & 6.781816 \\ \text { S } & 7.083600 & 7.083600 & 5.588032 \\ \text { H } & 6.294149 & 4.929413 & 6.151002 \\ \text { H } & 7.873051 & 9.237787 & 6.151002 \\ \text { S } & 9.066518 & 8.003056 & 15.684493 \\ \text { S } & 7.083600 & 7.083600 & 17.869868 \\ \text { C } & 8.400730 & 6.600584 & 16.676084 \\ \text { H } & 9.237787 & 6.294149 & 17.306898 \\ \text { C } & 5.766470 & 7.566616 & 16.676084 \\ \text { S } & 8.002705 & 5.100545 & 15.684128 \\ \text { C } & 7.566586 & 8.400604 & 14.692304 \\ \text { S } & 5.100682 & 6.164144 & 15.684493 \\ \text { H } & 4.929413 & 7.873051 & 17.306898 \\ \text { S } & 6.164495 & 9.066655 & 15.684128 \\ \text { C } & 6.600614 & 5.766596 & 14.692304 \\ \text { S } & 7.083600 & 7.083600 & 13.497800 \\ \text { H } & 6.294240 & 4.929612 & 14.061285 \\ \text { H } & 7.872960 & 9.237588 & 14.061285 \\ \text { E } & & & \\ \text { EB+HF - LYP })= & -5087.84316105 & \end{array}$

